\title{
REPETITIVE LEARNING OF BACKSTEPPING CONTROLLED NONLINEAR ELECTROHYDRAULIC MATERIAL TESTING SYSTEM ${ }^{1}$
}

\author{
Seunghyeokk James Lee ${ }^{2}$, Tsu-Chin Tsao \\ Mechanical and Aerospace Engineering Department \\ University of California Los Angeles, Los Angeles, CA 90095
}

\begin{abstract}
This paper develops repetitive and iterative learning control design and analysis for back stepping controlled nonlinear systems. To precisely track periodic or finite duration trajectories for nonlinear systems, back stepping control is first designed to render closed loop stability and, in theory, asymptotic tracking performance. However, due to the sensitivity to the unmodelled dynamics plant variations, asymptotic tracking performance is usually not feasible. Thus, repetitive or learning control is applied over the back stepping controlled system to restore asymptotic tracking performance. This approach is applied to an electrohydraulic material testing system, in which the material specimen present substantial nonlinear force and displacement relationship. It will be shown that the back stepping control employs both feedback and feedforward actions to render linearized I/O plant and thus the outer loop repetitive and learning control design can be based on the compensated linear system. Experimental results will be given to demonstrate the effectiveness of the proposed approach.
\end{abstract}

Keywords: Electro-hydraulic systems, hydraulic actuators, learning control, nonlinear control system.

\section{INTRODUCTION}

Mechanical properties are identified by material testing operations such as the impact test, creep test, tensile compression test and fatigue test, etc. Among others, the tensile/compression test is the most frequently performed test. The tensile/compression test measures the resistance of a material by statically or dynamically applied force. A testing material is placed between the upper grip and the lower grip. A force, called load, is then applied by an actuator. Sensors are used to measure the force, strain, and net displacement of the specimen, respectively. Electrohydraulic actuators are widely used in material testing operations because of its ability to generate a large force with wide bandwidths. In most cases, they are operated under a simple P or PI closed loop control to establish stability. Manual tuning of the controller gains are usually exercised for a specific test, depending on the specimen stiffness, the testing profile and ranges. Typical modes of material testing control include the load (force or differential pressure) control, strain control, and displacement control of the specimen.

In dynamic testing where the specified profile is dynamic, it is desirable to closely track the desired profile to fulfill the testing design specification. The nonlinearities presented in the system prevent the simple linear feedback control to achieve good tracking performance. Several papers on the control application of material testing machines have been published by Lee and Srinivasan $(1989,1990)$. Their linear model based controller performs well only in low frequency, and low velocity range testing, where the system is assumed to be close to linear. An adaptive PID control scheme was applied to the material testing machine by Hinton and Clarke (1994). Their adaptive PID controller is able to correct variations caused by the change in specimen stiffness. The adaptive PID controller was performed on metal specimens, which have linear stiffness. No tests were performed on specimens that had nonlinear stiffness, such as an elastomer.

While the systematic approach to integrator backstepping control for nonlinear systems have been introduced in the book of Kristic et al. (1995), there have been rare publications addressing its applications (Lin and Kanellakopoulos, 1997) and implementation issues (Bupp et al. 1998, Alleyne and Liu, 2000). The main implementation difficulties stem from the huge control input and chattering often occurs in the transient stage, since the initial state of the controller is a guesswork. The system may generate high frequency ringing or may not even be stable, let alone asymptotic tracking performance.

\footnotetext{
${ }^{1}$ This research was supported in part by Moog Inc., East Aurora, New York.

${ }^{2}$ This co-author was a visitor at the University of California Los Angeles while as a Mater of Science student at the University of Illinois at Urbana-Champaign
} 
Recently, Lee and Tsao (2002) designed and implemented a back stepping control for the displacement control of an electrohydraulic actuated material testing systems. The nonlinearity modeled in that system was the nonlinear stiffness of the specimen under testing. With careful modelling, identification, and some techniques to treat the transient response problem, the back stepping control implementation was able to achieve a tracking performance that brings the tracking error magnitude to less than $2 \%$ of the reference signal.

The asymptotic tracking performance achieved by the back stepping control theory, however, relies on the accuracy of the plant model accuracy. This is because the back stepping control applies a feedforward compensation to the back stepping compensated closed loop system. In order to achieve robust asymptotic tracking performance for the class of signal generated by a dynamic system, the internal model principle suggests that it is necessary to include the signal dynamic model in the feedback control loop. However, it is not obvious as how the internal model should be inserted into the backstepping control system to achieve the robust asymptotic tracking performance.

This paper addresses the above question of designing and integrating repetitive control and backstepping control for nonlinear systems. The repetitive control is one of the more challenging internal model principle type control problems since the internal model is of infinite order in continuous time domain and of high order in the discrete-time domain. Furthermore, it can be shown that the method of designing repetitive control for tracking periodic reference signals can also be applied to learning control analogously to track finite duration reference signals through cycle by cycle iterative compensation.

The rest of this paper is organized as follows. Section 2 summarizes the system model and the backstepping control of the nonlinear electrohydraulic material testing system under consideration. Section 3 gives the main results of the backstepping control for the material testing system. Section 4 proposes two ways to inject repetitive control signal to the backsteping control system, one from the control input, and the other from the reference input signal. The conditions for the stability and asymptotic tracking performance will be given. Section 5 presents the backstepping and repetitive control implementation and experimental results followed by the conclusion in the final section.

\section{EXPERIMENTAL SYSTEM AND MODEL}

The electrohydraulic materials testing system mainly consists of a double acting equal area hydraulic actuator mounted on the top of a specially made material testing frame. The design constraints for the material testing frame were derived for elastomer testing application. The actuator of force area is 0.56 in 2 is controlled by a flapper nozzle type two-stage mechanical spring feedback servovalve with $7.5 \mathrm{gpm}$ rated flow rate at 1000 psi pressure drop across the valve. Thus, the maximum static force is $1,680 \mathrm{lbf}$ and the maximum velocity of the actuator is approximately $2.34 \mathrm{~m} / \mathrm{sec}$ at 3000 psi supply pressure.

The sensor instrumentation include a laser displacement encoder measuring the actuator displacement, a load cell measuring the force between the frame and the material specimen, a Linear Variable Differential Transformer (LVDT) measuring the servovalve spool position, and two pressure transducers, measuring the pressure on each side of the actuator. The pressure difference was used to obtain the force from the actuator.

The elastomer, a number of natural and synthetic linear polymers, has substantial nonlinearity between force and displacement. The nonliner force versus displacement data was first obtained experimentally and then curve fitted with parameterized functions to different degree of accuracy so that the effect of this nonlinearity on the control sstem performance can be evaluated. In the first case, a $4^{\text {th }}$ order polynomial is used as shown in Figure 1. In the second case three piecewise linear segments were used as shown in Figure 2. In the third case, a simple straight line is used, as shown in Figure 3.

The nonlinear model of the electrohydraulic system has been well studied. Kim and Tsao (2000) derived the linearization of the nonlinear model as an $8^{\text {th }}$ order transfer function consisting of a cascaded system $3^{\text {rd }}$ order actuator model and $5^{\text {th }}$ order servovalve model and showed that the linearized $8^{\text {th }}$ order model fits the experimental frequency response obtained from the same system used in this paper closely up to $1000 \mathrm{~Hz}$. Considering the frequency range of interest in material testing, a reduced first order servo valve model, which fits well with the full order model well up to $100 \mathrm{~Hz}$, and the $3^{\text {rd }}$ order actuator model will be used for the control system design in this paper. The system model is given as follows:

$$
\begin{aligned}
& \dot{x}_{1}=x_{2} \\
& \dot{x}_{2}=-a_{1} x_{2}-f\left(x_{1}\right)+a_{3} x_{3} \\
& \dot{x}_{3}=-a_{4} x_{2}+a_{5} x_{4} \\
& \dot{x}_{4}=-a_{6} x_{4}+a_{7} u
\end{aligned}
$$

where

$$
\begin{aligned}
& a_{1}=\frac{b}{M}, \quad a_{2}=\frac{1}{M}, \quad a_{3}=\frac{A}{M}, \quad a_{4}=\frac{4 \beta_{e} A}{V_{t}}, \\
& a_{5}=\frac{4 \beta_{e} K}{V_{t}}, \quad a_{6}=\frac{1}{\tau}, \quad a_{7}=\frac{K}{\tau}, \\
& f\left(x_{1}\right)=a_{2} \varphi\left(x_{1}\right) \\
& x 1: \text { displacement of actuator } \\
& x 2: \text { velocity of actuator }
\end{aligned}
$$


$x 3$ : difference of pressure

$x 4$ : displacement of spool

$M:$ mass of double ended actuator and clevis

$b:$ damping coefficient of the actuator

$A:$ actuator ram area

$V t$ : total actuator volume

$\beta$ : effective bulk modulus

$K_{-}$: flow coefficient caused by pressure

$\tau$ : time constant

$K$ : amplifier gain

$\varphi\left(x_{1}\right)$ : Stiffness of the specimen

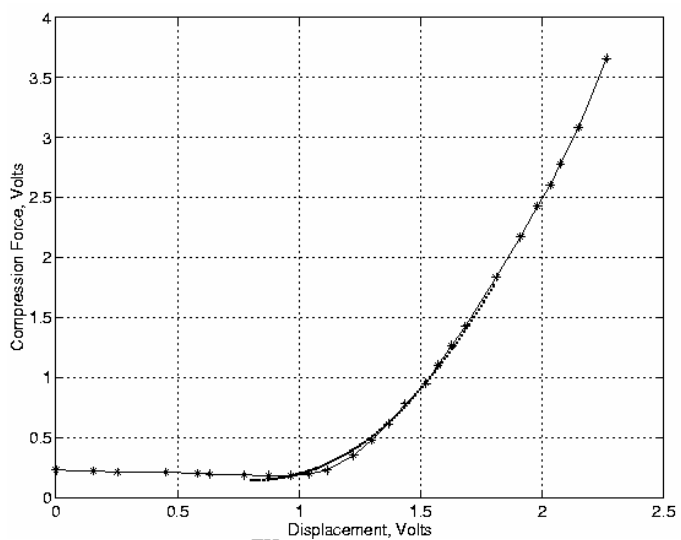

Fig. 1. $4^{\mathrm{TH}}$ order curve fit model.

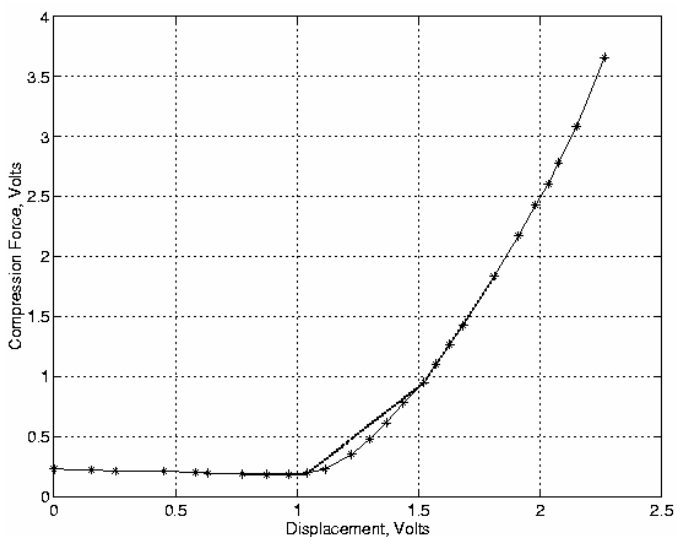

Figure 2. piecewise (3 segments) linear curve fit model.

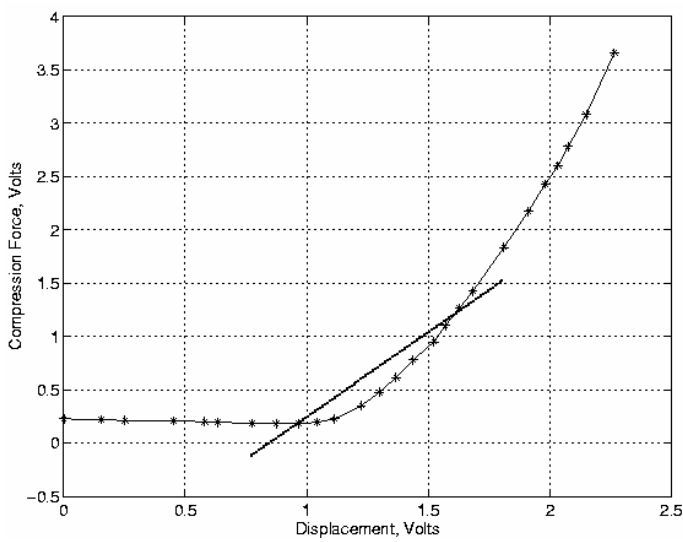

Figure 3. Single Linear Segment Model

\section{BACKSTEPPING CONTROL DESIGN}

The backstepping signal synthesis for the nonlinear system in (1) has been given in detail in Lee and Tsao (2002). Without repeating the design procedure, the resulting closed loop control system is given below:

$$
\left[\begin{array}{l}
\dot{z}_{1} \\
\dot{z}_{2} \\
\dot{z}_{3} \\
\dot{z}_{4}
\end{array}\right]=\left[\begin{array}{cccc}
-C_{1} & 1 & 0 & 0 \\
-1 & -C_{2} & 1 & 0 \\
0 & -1 & -C_{3} & 1 \\
0 & 0 & -1 & -C_{4}
\end{array}\right]\left[\begin{array}{l}
z_{1} \\
z_{2} \\
z_{3} \\
z_{4}
\end{array}\right]
$$

In (2), $z_{1}$ is the negative of the tracking error yr- $y$. The controller consists of nonlinear feedback and linear feedforward compensation as follows:

$$
\begin{aligned}
& u(t)=f\left(x_{1}, x_{2}, x_{3}, x_{4}\right)+ \\
& \beta_{0} y_{r}+\beta_{1} \dot{y}_{r}+\beta_{2} \ddot{y}_{r}+\beta_{3} \dddot{y}_{r}+\beta_{4} \dddot{y}_{r}
\end{aligned}
$$

The coefficients $\beta_{\mathrm{i}}$ of the feedforward terms form the characteristic polynomial of (2):

$\beta_{0}=1+C_{1} C_{2}+C_{1} C_{4}+C_{3} C_{4}+C_{1} C_{2} C_{3} C_{4}$

$\beta_{1}=2 C_{1}+C_{2}+C_{3}+2 C_{4}+C_{1} C_{3} C_{4}+C_{1} C_{2} C_{3}+C_{2} C_{3} C_{4}$

$\beta_{2}=3+C_{1} C_{2}+C_{1} C_{3}+C_{1} C_{4}+C_{2} C_{3}+C_{2} C_{4}+C_{3} C_{4}$

$\beta_{3}=C_{1}+C_{2}+C_{3}+C_{4}$

$\beta_{4}=1$

The feedback control portion of the control in (3) involves terms from the specimen nonlinearity $\varphi\left(x_{1}\right)$ and derivatives, to be precise, the first and second order derivative of $\varphi\left(x_{1}\right)$. These derivatives are needed because there are two integrators between the nonlinear term $\varphi\left(x_{1}\right)$ and the control signal, in view of the plant model in Eq. (1).

\section{REPETITIVE AND ITERATIVE LEARNING CONTROL DESIGN}

To understand further where the control signal enter the closed loop system, it is noted that the fourth state in (2), before the control in (3) is applied is in the following form:

$$
\dot{z}_{4}=\alpha u+\ldots
$$

where those terms not shown are not functions of the control input and

$$
\alpha=a_{3} a_{5} a_{7}
$$

Thus, the control input $u$ may be modified to inject a new term from the repetitive learning control: 


$$
u(t)+\frac{1}{\alpha} u_{r e p}(t) \rightarrow u(t)
$$

where $u_{\text {rep }}(t)$ is the outer loop repetitive or learning control signal. By adding this, the closed loop backstepping control system as seen by the repetitive control input and the relation to the tracking error output is a stable linear system:

$$
\begin{aligned}
& {\left[\begin{array}{l}
\dot{z}_{1} \\
\dot{z}_{2} \\
\dot{z}_{3} \\
\dot{z}_{4}
\end{array}\right]=\left[\begin{array}{cccc}
-C_{1} & 1 & 0 & 0 \\
-1 & -C_{2} & 1 & 0 \\
0 & -1 & -C_{3} & 1 \\
0 & 0 & -1 & -C_{4}
\end{array}\right]\left[\begin{array}{l}
z_{1} \\
z_{2} \\
z_{3} \\
z_{4}
\end{array}\right]+\left[\begin{array}{l}
0 \\
0 \\
0 \\
1
\end{array}\right] u_{\text {rep }}} \\
& e=-\left[\begin{array}{llll}
1 & 0 & 0 & 0
\end{array}\right]\left[\begin{array}{llll}
z_{1} & z_{2} & z_{3} & z_{4}
\end{array}\right]^{T}
\end{aligned}
$$

Thus the transfer function for (8) can be used for designing repetitive or learning control:

$$
G(s)=\frac{-e(s)}{u_{\text {rep }}(s)}=\frac{1}{\beta_{4} s^{4}+\beta_{3} s^{3}+\beta_{2} s^{2}+\beta_{1} s+\beta_{0}}
$$

In iterative learning control, it is usually the reference input trajectory that is modified cycle by cycle. In some situation the control input signal may not be accessible for modification while the programmable external reference signal is. Thus, it is desirable to inject the repetitive or learning compensation into the reference signal. To achieve this, a compensation term may be added to the reference input in (3) without changing the derivatives :

$$
y_{r}(t)+y_{r_{-} r e p}(t) \rightarrow y_{r}(t)
$$

The input-output transfer function for this case is

$$
G(s)=\frac{-e(s)}{y_{\text {rep }}(s)}=\frac{\beta_{0}}{\beta_{4} s^{4}+\beta_{3} s^{3}+\beta_{2} s^{2}+\beta_{1} s+\beta_{0}}
$$

The linear model in (9) or (11) can then be used for the repetitive or learning control design. The prototype robust repetitive control in the transformed domain is as follows (Tsao and Tomizuka, 1994):

$$
\frac{u_{r e p}(s)}{e(s)}=\frac{K_{r} M(s) e^{-S T_{p}}}{1-Q(s) e^{-S T_{p}}}
$$

where $T p$ is the period of the signal, $\mathrm{M}(\mathrm{s})$ and $\mathrm{Q}(\mathrm{s})$ are design parameters in transfer function forms and $\mathrm{Kr}$ is a scalar gain. If the closed loop system formed by (12) is asymptotically stable, the error asymptotically converges to zero. A sufficient condition for asymptotic stability is (Tsao and Tomizuka, 1994)

$$
\left|1-K_{r} M(j \omega) G(j \omega)\right|<\frac{1}{|Q(j \omega)|}, \quad \forall \omega
$$

where $G(s)$ is the transfer function from (10) or (11) depending on where the signal is injected. Note that the plant dynamics $G(\mathrm{~s})$ used for the repetitive control design depends only on the back stepping closed loop dynamics. Unmodeled dynamics will perturb the transfer function $G(s)$ as seen by the repetitive control. If (12) is satisfied for the perturbed plant, which includes the unmodeled dynamics, robust stability and asymptotic tracking performance is achieved. Equations (11) and (12) can also be written in the discrete-time domain similarly and are used more often since the control is implemented by a digital computer anyway.

\section{EXPERMENTAL RESULTS}

\subsection{Backstepping Control}

While the backstepping control input was designed in continuous time domain, the controller was implemented by a digital signal processor at a sampling frequency of $5000 \mathrm{~Hz}$. A major difficulty encountered in implementing the backstepping controller was the very large and chattering control input signals during the transition state. The computed control signal could easily go over the maximum input limits set to safeguard the plant. In implementation the control saturation could cause control signal chattering between the saturation limit. Figure 4 shows an example of such situation.

To avoid input saturation and chattering, reduction on the design parameters $\mathrm{Ci}^{\prime} s$ were implemented during the transient stage, which in effect corresponds to reducing gains on the state's feedback terms. The feedforward part remains unchanged to force fast transient tracking errors convergence without causing saturation. As soon as the control input reaches near steady state, the reduction gains on the feedback part are removed. Thus any disturbance coming in after this will be rejected at the designed rate.

This method ensures that applying the reduction factors would not have any affect upon the tracking performance in the steady state since the feedforward part is supposed to take care of the major part of the tracking and is not altered during the transient stage. In the experiment, the displacement reference signal was a $30 \mathrm{~Hz}$ sine wave between 0.8 and 1.8 volts. Figure 5 shows the experimental result using the most accurate nonlinear specimen model in Case 1. The design parameters were $\mathrm{C} 1=900, \mathrm{C} 2=1000$, $\mathrm{C} 3=1000$ and $\mathrm{C} 4=1000$. The tracking error was a little less than $\pm 0.05(\mathrm{~V})$. The significance of the nonlinear term and its derivatives is demonstrated by using the piecewise linear and linear models in the Case 2 and 3 respectively. Figure 6 shows the 
experimental tracking error for the piecewise linear model in Case 2 function. The best tracking performance obtained was \pm 0.1 (V). While the piecewise linear function appears to agree with the nonlinear curve well as shown in Figure 2, its derivatives do not match that well. These high order derivative terms cause the tracking performance degradation and illustrate their significance in the backstepping control. The experimental results for the simple linearization model, Case 3 , is shown in Figure 7. It was difficult to stabilize the plant with this controller, but, by re-tuning the control parameters Ci's, the tracking error was reduced to \pm $0.24(\mathrm{~V})$.

The nonlinear term, $\varphi\left(x_{1}\right)$ was replaced by the measured force feedback signal from the load cell in the implementation of Case 1. The derivative terms from the nonlinear model were maintained in the control implementation. Since the force feedback represents a direct measurement of the nonlinear force, a better tracking performance is expected. Indeed, the tracking error was further reduced to \pm 0.008 (V), as shown in Figure 8. This result again suggests the importance of an accurate nonlinear model or measurement in the backstepping control.

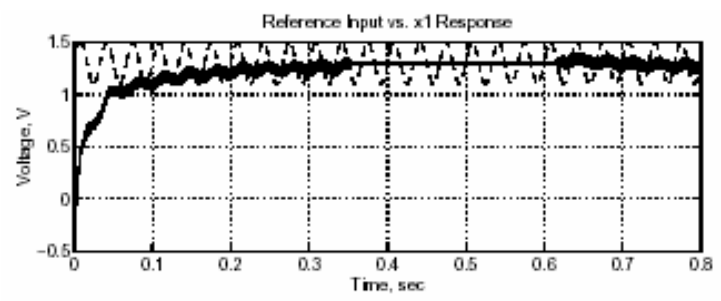

Figure 4. Control Chattering Example

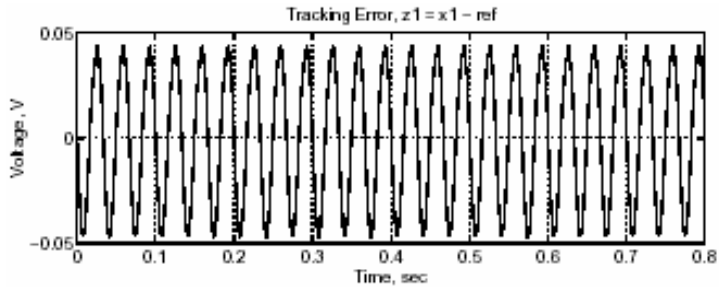

Figure 5. Tracking Error of Case 1 Experiment

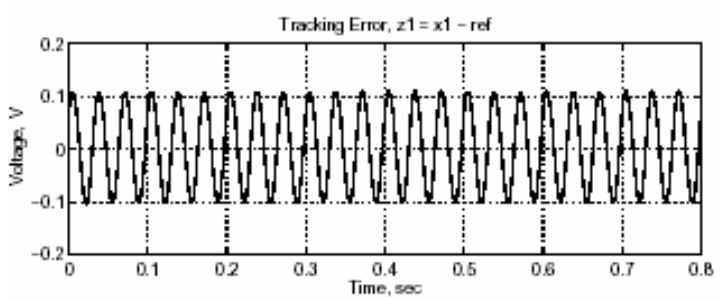

Figure 6. Tracking Error of Case 2 Experiment

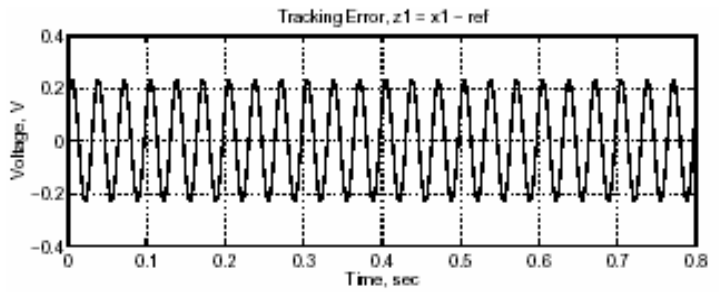

Figure 7. Tracking Error of Case 3 Experiment
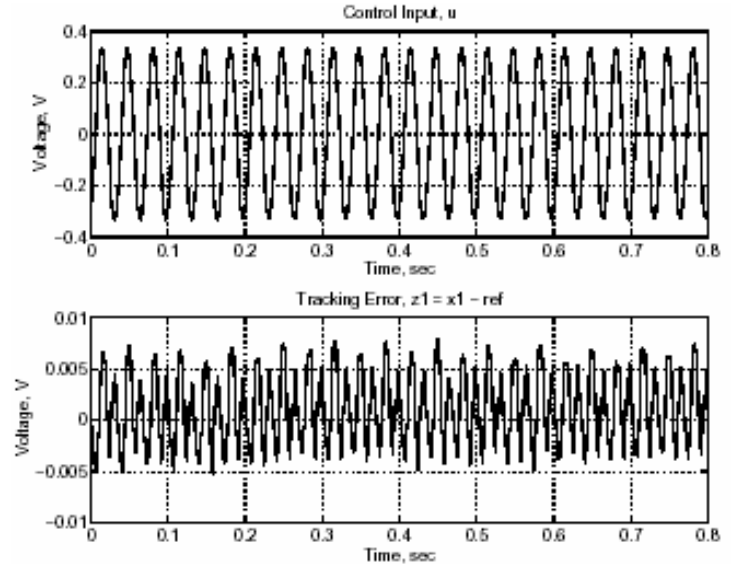

Figure 8: Control And Tracking Error Signal of Case 1 Experiment with Force Feedback

\subsection{Repetitive Learning Control}

After the repeated testing, the nonlinear specimen had changed somewhat. Then the above control performance no longer holds. The tracking error with force feedback had increased from \pm 0.008 to \pm 0.08 (V). Figure 9 shows the tracking error for such a situation. To recover the control performance due to plant variations, repetitive control approach as discussed in the previous section was implemented.

Three prototype repetitive controllers were designed for modifying the reference input signal as in (10) based on the backstepping compensated plant model in (11). The controller in (12) was implemented in the discrete-time domain with the following parameters:

Case $\mathrm{A}: \mathrm{M}=1, \mathrm{Kr}=0.01$,

$\mathrm{Q}=\mathrm{Q} 1=0.25 z+0.5+0.25 z^{-1}$

Case $\mathrm{B}: \mathrm{M}=1, \mathrm{Kr}=0.02$,

$\mathrm{Q}=\mathrm{Q} 2=\left(0.25 z+0.5+0.25 z^{-1}\right)^{2}$

Case $\mathrm{C}: \mathrm{M}=1, \mathrm{Kr}=0.02$,

$\mathrm{Q}=\mathrm{Q} 4=\left(0.25 z+0.5+0.25 z^{-1}\right)^{4}$

These control parameters were chosen based on the stability condition given in (13). Figure 10, where the right hand side of Eq. (13) is shown in solid lines for the three $\mathrm{Kr}$ values and the left hand side of Eq. (13) is plotted in the dashed lines for the three Q's, shows that the inequality in (13) is narrowly satisfied for each of the three cases.

In the implementation for Case A, the system was marginally stable and sometimes became unstable. Thus, no data is included. The tracking error for the implementation of Case B was $\pm 0.01(\mathrm{~V})$ as shown in Figure 11. Finally, the tracking error for Case C was about $\pm 0.008(\mathrm{~V})$, as shown in Figure 12. Thus, the tracking error was recovered from $0.08(\mathrm{~V})$ to $0.006(\mathrm{~V})$ by the repetitive controller. 
Traking Error, $z 1=x 1-$ rof

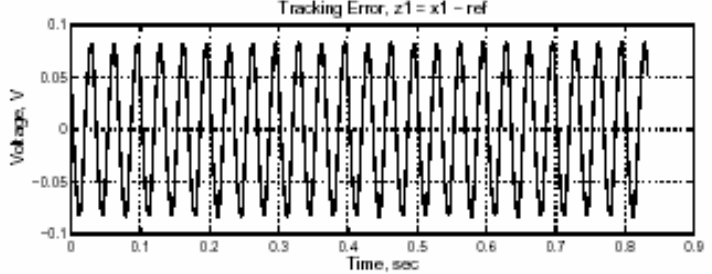

Figure 9 Tracking Error of the Force Feedback Backstepping Control After System Variations

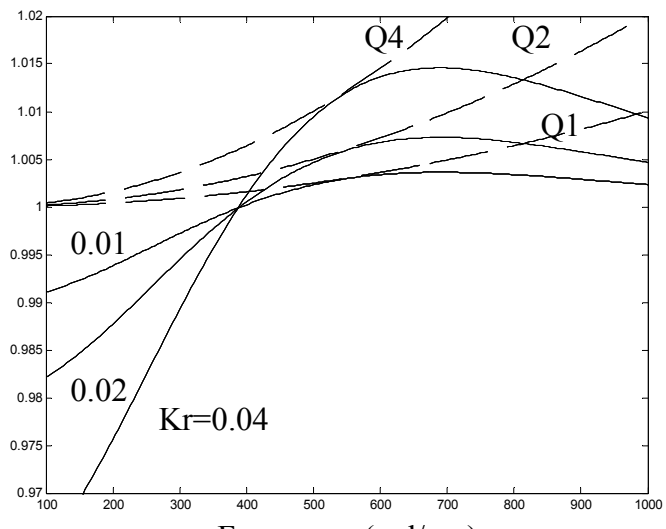

Frequency ( $\mathrm{rad} / \mathrm{sec})$

Figure 10. Inequality Illustration for the Repetitive Control Design

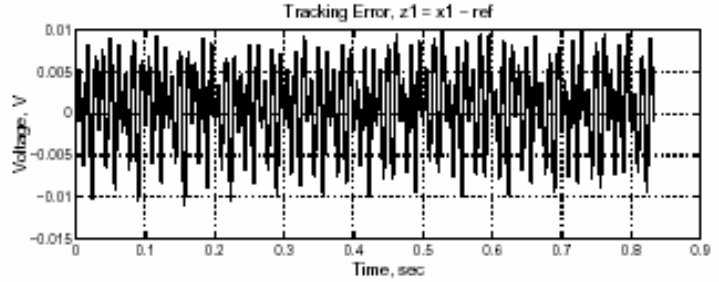

Figure 11. Tracking Error of Case B Experiment

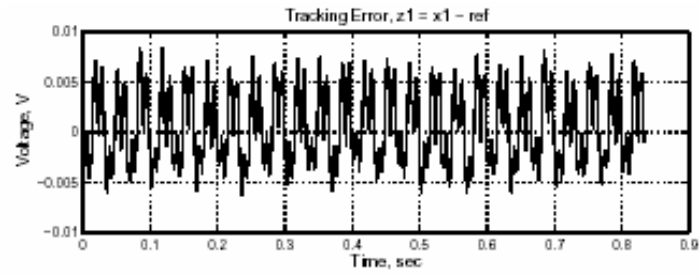

Figure 12. Tracking Error of Case C Experiment

\section{CONCLUSION}

The backstepping controller was designed and implemented to control a nonlinear electrohydraulic actuated material testing machine. Commonly occurred large control input and chattering in the transition state are avoided by applying reduction factors in the feedback terms during the initial transient stage. Since the nonlinear terms and derivatives propagate in the backstepping control, it is important to have an accurate nonlinear model for achieving superior control performance. Asymptotic tracking performance of backstepping control system is sensitive to model variations. To account for the model variations, repetitive control was designed for the backstepping controlled closed loop system.
With adequate injection points of the repetitive compensation signal to the backstepping control system, the asymptotic tracking can be recovered. Experimental results on the backstepping and repetitive control implementation on the electrohydraulic material testing system shows the effectiveness of the control approach developed herein.

\section{REFERENCES}

Alleyne A. G. and R. Liu, 2000, "Systematic Control of a Class of Nonlinear Systems with Application to Electrohydraulic Cylinder Pressure Control ", IEEE Control Systems Technology, Vol. 8, No. 4, pp. 623-634.

Bupp, R. T., D.S. Bernstein and V.T. Coppola, 1988, "Experimental Implementation of Integrator Backstepping and Passive Nonlinear Controllers on the RTAC Testbed ", International Journal of Robust and Nonlinear Control, Vol. 8, pp. 435457.

Hinton C. E. and D.W. Clarke, 1994, "Control of Servo-Hydraulic Materials-Testing Machines", Ph.D. Dissertation, University of Oxford.

Kim, D. H. and T-C. Tsao, 2000, "A Linearized Electrohydraulic Servovalve Model for Valve Dynamics Sensitivity Analysis and Control System Design",ASME Journal of Dynamic Systems and Measurement, and Control, Vol. 122, pp. 179-187.

Kristic, M., I. Kanelloakopoulos, and P. Kokotovic, 1995, "Nonlinear and Adaptive Control Design," John Wiley \& Sons, Inc.

Lee, H. S. and T-C. Tsao, 2002, "Nonlinear Backstepping Control of An Electrohydraulic Material Testing System," Proceedings of the American Control Conference, Anchorage, AK, pp. 4625-4630.

Lee, S. R. and K. Srinivasan, 1989, "On-Line Identification of Process Models in Closed Loop Material Testing ", ASME Journal of Dynamic Systems, Measurement, and Control, Vol. 111, No. 2, pp. 172-179.

Lee, S. R. and K. Srinivasan, 1990, "Self-Tuning Control Application to Closed Loop Servohydraulic Material Testing”, ASME Journal of Dynamic Systems, Measurement, and Control, Vol. 112, pp. 680-689.

Lin J. S. and I. Kanellakopoulos, 1997, "Nonlinear Design of Active Suspensions", IEEE Control Systems Magazine, Vol. 17, pp. 45-59.

Tsao, T. C. and M. Tomizuka, 1994, "Robust Adaptive and Repetitive Digital Tracking Control and Application to a Hydraulic Servo for Noncircular Machining," ASME Journal of Dynamic Systems, Measurement, and Control, 116:1, 24-32, 1994. 